\title{
UNIFORM ALGEBRAS INVARIANT UNDER EVERY HOMEOMORPHISM
}

\author{
ALEXANDER J. IZZO \\ Dedicated to James Munkres on the occasion of his 80th birthday
}

\begin{abstract}
For a broad class of spaces $X$, we show that $C(X)$ is the only uniform algebra on $X$ that is invariant under every self-homeomorphism of $X$. This class of spaces contains the manifolds-with-boundary and the finite simplicial complexes. We also give examples showing that the result fails for CW complexes.
\end{abstract}

\section{INTRODUCTION}

This paper addresses the following question: In the complex form of the StoneWeierstrass theorem, if the hypothesis that the algebra is self-adjoint is omitted and replaced by the hypothesis that the algebra is invariant under every selfhomeomorphism of the underlying space, does the conclusion of the theorem still hold?

For $X$ a compact Hausdorff space, let $C(X)$ denote the algebra of all complexvalued continuous functions on $X$. (Throughout the paper all spaces will tacitly be required to be Hausdorff except where noted otherwise.) Since uniformly closed subalgebras of $C(X)$ that contain the constants and separate points are called uniform algebras, our question can be restated as follows: If $A$ is a uniform algebra on a compact space $X$ and $A$ is invariant under every self-homeomorphism of $X$, must $A=C(X)$ ? It turns out that the answer is no in general but is yes for $X$ belonging to a broad class of spaces.

Before describing in more detail the results to be presented, we discuss the author's motivation for considering the above question. Inspired by a question raised by Ronald Douglas in connection with his work on a conjecture in operator theory due to William Arveson, the author considered uniform algebras invariant under group actions in [9,10]. To say that a uniform algebra $A$ on $X$ is invariant under the action of a group $G$ on $X$ means that the function $x \mapsto f(g x)$ is in $A$ for every element $g \in G$ whenever $f$ is in $A$, where $g x$ denotes the image of the pair $(g, x)$ under the action. As usual, topological group actions will be taken to be continuous and Lie group actions to be smooth, i.e., of class $C^{\infty}$. Among the results in 9 ] is the following.

Received by the editors May 14, 2012 and, in revised form, November 14, 2012.

2010 Mathematics Subject Classification. Primary 46J10; Secondary 22F50, 32A65, 54C35, 54H15, 57P99, 57S99.

This paper was presented to the American Mathematical Society in preliminary form on April 9, 2011 under the title Function algebras invariant under every self-homeomorphism. 
Theorem 1.1. Let $A$ be a uniform algebra on a smooth manifold $M$. Suppose that the maximal ideal space of $A$ is $M$ and that the algebra $A$ is invariant under the action of a Lie group that acts transitively on $M$. Then $A=C(M)$.

In view of this theorem, the author conjectured [9] that the same result holds more generally for a uniform algebra on an arbitrary compact space invariant under a transitive action by an arbitrary topological group. The precise statement of the conjecture is as follows.

Conjecture 1.2. Let $A$ be a uniform algebra on a compact space $X$. Suppose that the maximal ideal space of $A$ is $X$ and that the algebra $A$ is invariant under the action of a topological group that acts transitively on $X$. Then $A=C(X)$.

Of course the conjecture applies only to uniform algebras on topological spaces on which the group of self-homeomorphisms acts transitively. So the question arises whether the conjecture can be generalized so as to apply to algebras on spaces without a transitive group of self-homeomorphisms. An obvious attempt at such a generalization would be to ask the following: If the action is not assumed transitive, but the orbit space of $X$ under the group $G$ coincides with the orbit space of $X$ under the action of the full self-homeomorphism group of $X$, must $A=C(X)$ ? The answer is trivially no. The disc algebra $A(D)$ is a counterexample. (The disc algebra $A(D)$ is the algebra of continuous functions on the closed unit disc $\bar{D}$ that are holomorphic on the open unit disc $D$.) This algebra is invariant under the action of the group of conformal self-maps of the disc, and the orbit space of that action coincides with the orbit space under the full self-homeomorphism group of the closed disc. It consists of two points: the open unit disc and the boundary of the disc.

The failure of this attempt at generalizing the conjecture suggests that we should consider what happens under the hypothesis that the algebra has as much invariance as possible. We are thus led to the question addressed in the present paper: If $A$ is invariant under every self-homeomorphism of $X$, must $A=C(X)$ ? As mentioned in the second paragraph, the answer is no in general but is yes for $X$ belonging to a broad class of spaces. We now introduce the class of spaces for which we will prove the answer is yes. We refer to these spaces as indexed manifold complexes or IM complexes for short.

We will reserve the word "manifold" to refer to boundaryless manifolds. Explicitly, by a manifold we will mean a Hausdorff space $M$ such that for some $m$ every point of $M$ has a neighborhood homeomorphic to an open set in $\mathbb{R}^{m}$. Note that we do not require our manifolds to be second countable or even paracompact. We will regard discrete spaces as manifolds of dimension zero. Manifolds-with-boundary will be explicitely referred to as such.

Definition 1.3. An indexed manifold complex, or IM complex, is a Hausdorff space $X$ and a collection $\left\{M_{\alpha}\right\}_{\alpha \in J}$ of disjoint manifolds whose union is $X$ such that

(1) the index set $J$ is well-ordered,

(2) for each $\alpha_{0} \in J$, the set $\bigcup_{\alpha \geq \alpha_{0}} M_{\alpha}$ is closed in $X$, and

(3) for each $\alpha_{0} \in J$, each point of $M_{\alpha_{0}}$ has a neighborhood $N$ in $M_{\alpha_{0}}$ such that every self-homeomorphism of $\bigcup_{\alpha \geq \alpha_{0}} M_{\alpha}$ that is the identity outside of $N$ extends to a self-homeomorphism of $X$ that sends each $M_{\alpha}$ to itself. 
It should perhaps be pointed out explicitly that it is assumed that each $M_{\alpha}$ is a subspace of $X$, that is, that the topology of each $M_{\alpha}$ is the subspace topology $M_{\alpha}$ inherits from $X$. We will generally not distinguish between an IM complex $\left(X,\left\{M_{\alpha}\right\}\right)$ and the underlying space $X$, and we will refer to a space $X$ as an IM complex if there is a collection $\left\{M_{\alpha}\right\}$ such that $\left(X,\left\{M_{\alpha}\right\}\right)$ is an IM complex. The reader who wishes to avoid well-ordered sets and transfinite induction may restrict attention to the case where the index set $J$ is finite. However, as will be discussed below, in full generality our main result contains a theorem of Walter Rudin concerning the class of spaces on which there exist nontrivial uniform algebras, but this is no longer the case if only finite (or countable) index sets are allowed.

We will also consider a slightly more general notion in which the collection $\left\{M_{\alpha}\right\}_{\alpha \in J}$ has a last member that is not required to be a manifold. Explicitly, we make that following definition.

Definition 1.4. An IM complex with exceptional set is a Hausdorff space $X$ and a collection $\left\{M_{\alpha}\right\}_{\alpha \in J}$ of disjoint subspaces whose union is $X$ such that

(1) the index set $J$ is well-ordered and has a largest element denoted by $\infty$,

(2) each $M_{\alpha}$ is a manifold except possibly for $M_{\infty}$, which is allowed to be completely arbitrary (and may be empty),

(3) for each $\alpha_{0} \in J$, the set $\bigcup_{\alpha \geq \alpha_{0}} M_{\alpha}$ is closed in $X$, and

(4) for each $\alpha_{0} \in J$, each point of $M_{\alpha_{0}}$ has a neighborhood $N$ in $M_{\alpha_{0}}$ such that every self-homeomorphism of $\bigcup_{\alpha \geq \alpha_{0}} M_{\alpha}$ that is the identity outside of $N$ extends to a self-homeomorphism of $X$ that sends each $M_{\alpha}$ to itself.

Examples of IM complexes are given in the next section. In particular, we show that every manifold-with-boundary is an IM complex and so is the underlying space of every finite-dimensional simplicial complex.

We can now state our main theorem.

Theorem 1.5. Let $X$ be a compact IM complex. If $A$ is a uniform algebra on $X$ that is invariant under every self-homeomorphism of $X$, then $A=C(X)$.

Note that here, in contrast to the situation in Theorem 1.1, no assumption is made regarding the maximal ideal space of $A$. In fact, the first part of the proof of Theorem 1.5, given in Section 4, consists of showing that the hypotheses imply that the maximal ideal space of $A$ is indeed $X$.

Definition 1.6. A self-homeomorphism $f: X \rightarrow X$ of the underlying space of an IM complex with exceptional set $\left(X,\left\{M_{\alpha}\right\}\right)$ is said to be a distinguished IM self-homeomorphism if $f\left(M_{\alpha}\right)=M_{\alpha}$ for each index $\alpha$.

Note that with this definition the last condition in the definition of IM complex and IM complex with exceptional set can be rephrased as asserting that each selfhomeomorphism of $\bigcup_{\alpha>\alpha_{0}} M_{\alpha}$ of the indicated type extends to a distinguished IM self-homeomorphism of $X$. In proving Theorem [1.5 we will use only that $A$ is invariant under every distinguished IM self-homeomorphism of $X$. Thus we have the following stronger result.

Theorem 1.7. Let $X$ be a compact IM complex. If $A$ is a uniform algebra on $X$ that is invariant under every distinguished IM self-homeomorphism of $X$, then $A=C(X)$. 
If we exclude zero-dimensional manifolds from the complex, then the theorem can be generalized to allow an exceptional set. For this we need to recall a standard, but not so well-known, notion introduced by Herbert Bear. The essential set for a uniform algebra $A$ on a space $X$ is the smallest closed subset $E$ of $X$ such that $A$ contains every continuous function on $X$ that vanishes on $E$. The proof of the existence of the essential set, along with other facts about the essential set, can be found in [2] or [4]. Note that $A$ contains every continuous function whose restriction to $E$ lies in the restriction $A \mid E$ of $A$ to $E$. Note also that $A=C(X)$ if and only if $E$ is the empty set.

Theorem 1.8. Let $\left(X,\left\{M_{\alpha}\right\}\right)$ be an IM complex with exceptional set and without manifolds of dimension zero. If $A$ is a uniform algebra on $X$ that is invariant under every distinguished IM self-homeomorphism of $X$ that is the identity on $M_{\infty}$, then the essential set for $A$ is contained in $M_{\infty}$.

In Section 3 we show, as mentioned above, that Theorem [1.5] does not hold for arbitrary compact spaces. Of course the question of on which spaces the theorem holds is related to the question of on which compact spaces there are no uniform algebras at all, other than $C(X)$. In the case of compact metric spaces, this question is completely answered by two theorems of Rudin. (Note that a space $X$ is said to be scattered if it contains no perfect subset or, equivalently, if every subset of $X$ has an isolated point.)

Theorem 1.9 (Rudin [14]). If $X$ is a compact space that contains a subspace homeomorphic to the Cantor set, then there is a uniform algebra $A$ on $X$ with $A \neq C(X)$.

Theorem 1.10 (Rudin [15]). If $X$ is a compact scattered space, then $C(X)$ is the only uniform algebra on $X$.

Since a compact metric space $X$ contains a Cantor set if and only if $X$ is not scattered, these two theorems show that there is a nontrivial uniform algebra on a compact metric space if and only if the space is not scattered. As discussed in Section 3, the existence of compact spaces for which Theorem 1.5 fails follows immediately from Theorem 1.9 and the existence of compact metric spaces with no nontrivial self-homeomorphisms. (We would like to thank James Kister for making us aware of the existence of such spaces and Jesus Araujo for informing us that these spaces are called rigid.) The algebras obtained from Theorem 1.9 are generally not defined on their maximal ideal spaces though, so this approach leaves open the question whether Theorem 1.5 would hold for arbitrary compact spaces under the additional hypothesis that the maximal ideal space $\mathfrak{M}_{A}$ of the algebra $A$ is $X$. However, we will give explicit examples satisfying this additional hypothesis. In fact, examples will be given of $\mathrm{CW}$ complexes for which the theorem fails both with and without the additional condition that $\mathfrak{M}_{A}=X$.

We will see in Section 5 that the IM complexes in which every manifold is zerodimensional are precisely the scattered spaces. From this it will follow that our main theorem contains, as a special case, the second of the theorems of Rudin quoted above.

Not surprisingly there are spaces that are not IM complexes for which Theorem 1.5 does hold. In fact, Kenneth Kunen [12] (see also [7, 13]) has shown that there exist nonscattered spaces on which there exist no nontrivial uniform algebras 
(and thus Rudin's characterization of the compact metric spaces on which there are no nontrivial uniform algebras does not extend to nonmetrizable spaces). It is immediate that such spaces cannot be IM complexes. In our context of uniform algebras invariant under every self-homeomorphism there are also much simpler examples. In particular, we will show that Theorem 1.5 holds for the Cantor set. This is not surprising, but it does provide an interesting counterpoint to the first theorem of Rudin quoted above.

It is a pleasure to dedicate this paper to James Munkres on the occasion of his 80th birthday. Munkres had a profound influence on the author at an early stage of his mathematical development. Indeed the author first learned many of the concepts used in this paper from him through his teaching and his books. In addition, it was from Munkres that the author first received guidance on mathematical exposition. Whatever merit there may be to this exposition is due in large part to his influence.

\section{EXAMPles of IM COMPLEXES}

Obviously every manifold is an IM complex. The following theorem provides many other examples of IM complexes and shows that the class of IM complexes is quite broad.

Theorem 2.1. If $X$ is any one of the following, then $X$ is an IM complex:

(a) a manifold-with-boundary;

(b) the underlying space of a finite-dimensional simplicial complex;

(c) a well-ordered set (with the order topology);

(d) the product of a well-ordered set with a manifold;

(e) the topologist's sine curve;

(f) the closed topologist's sine curve;

(g) the one-point compactification of a locally compact IM complex;

(h) a union of two manifolds $A$ and $B$, each of which is closed in $X$ and such that $A \cap B$ is a submanifold of each of $A$ and $B$.

Proof. (a) Suppose that $X$ is an $n$-manifold-with-boundary. Let $M_{1}$ be the interior of $X$, and let $M_{2}$ be the boundary of $X$. The conditions in the definition of an IM complex other than (3) are obviously satisfied. In the case when $n=1$, it is also obvious that condition (3) is satisfied as well, so we will assume $n>1$. Given a point $p$ in $M_{2}$, let $(U, \varphi)$ be a coordinate chart centered at $p$ with $\varphi(U) \supset$ $[-1,1]^{n-1} \times[0,1]$ and $\varphi(p)=0$. Let $N=\varphi^{-1}\left((-1,1)^{n-1} \times\{0\}\right)$. Then $N$ is a neighborhood of $p$ in $M_{2}$. Now let $h$ be an arbitrary self-homeomorphism of $M_{2}$ that is the identity outside of $N$. Then $h$ induces, via $\varphi$, a self-homeomorphism $g$ of $[-1,1]^{n-1} \times\{0\}$ that is the identity outside of $(-1,1)^{n-1} \times\{0\}$. Let $C$ be the cone over $[-1,1]^{n-1} \times\{0\}$ with vertex $(0, \ldots, 0,1)$. Denote the point $\left(x_{1}, \ldots, x_{n-1}\right)$ in $\mathbb{R}^{n-1}$ by $x^{\prime}$, and denote the origin of $\mathbb{R}^{n-1}$ by $0^{\prime}$. Define a map $l: C \rightarrow C$ by

$$
l\left(x^{\prime}, x_{n}\right)= \begin{cases}\left(1-x_{n}\right) g\left(x^{\prime} /\left(1-x_{n}\right)\right)+\left(0^{\prime}, x_{n}\right) & \text { for } x_{n} \neq 1 \\ \left(0^{\prime}, 1\right) & \text { for } x_{n}=1\end{cases}
$$

Then $l$ is a self-homeomorphism of $C$ that is the identity outside of the set $C^{0}=$ $\left\{(1-t)\left(x^{\prime}, t\right): x^{\prime} \in(-1,1)^{n-1}, 0 \leq t<1\right\}$, and $l$ extends $g$. Thus $l$ induces a self-homeomorphism $\tilde{l}$ on $\varphi^{-1}(C)$ that agrees with $h$ on $\varphi^{-1}\left([-1,1]^{n-1} \times\{0\}\right)$ and 
is the identity outside of $\varphi^{-1}\left(C^{0}\right)$. Now define $H: X \rightarrow X$ by

$$
H(x)= \begin{cases}\tilde{l}(x) & \text { for } x \in \varphi^{-1}(C) \\ x & \text { for } x \in X \backslash \varphi^{-1}\left(C^{0}\right)\end{cases}
$$

Note that $H$ is well defined and is a homeomorphism. Furthermore, $H$ extends $h$.

(b) Let $K$ be a simplicial complex of dimension $n$, and let $X$ be the underlying space $|K|$ of $K$. Let $J=\{0,1, \ldots, n\}$, ordered so that $n<n-1<\ldots<1<0$. Let $M_{j}$ be the union of the open simplices in $K$ of dimension $j$. Note that the sets in condition (2) of the definition of IM complex are the underlying spaces of the $p$-skeletons $K^{p}$ of $K$. Thus all that needs to be proved is condition (3).

Note that if $g: S^{n-1} \rightarrow S^{n-1}$ in any self-homeomorphism of the unit sphere $S^{n-1}$ in $\mathbb{R}^{n}$, then the map $G: B^{n} \rightarrow B^{n}$ given by

$$
G(x)= \begin{cases}\|x\| \cdot g(x /\|x\|), & x \neq 0 \\ 0, & x=0\end{cases}
$$

is a self-homeomorphism of the closed unit ball $B^{n}$ in $\mathbb{R}^{n}$ that extends $g$. Since every $n$-simplex $\sigma$ is homeomorphic to $B^{n}$ by a homeomorphism that maps the boundary $\operatorname{Bd} \sigma$ of $\sigma$ onto $S^{n-1}$, it follows that every self-homeomorphism of $\operatorname{Bd} \sigma$ extends to self-homeomorphisms of $\sigma$. From this it follows that every self-homeomorphism of $\left|K^{p}\right|$ that maps each simplex of $K^{p}$ onto itself extends to a self-homeomorphism of $\left|K^{p+1}\right|$, and hence by induction, extends to a self-homeomorphism of the full polyhedron $|K|=X$ taking each simplex to itself. Consequently, for each $j$, if $p$ is a point of $M_{j}$ and $N$ is the open simplex containing $p$, then every self-homeomorphism of $\left|K^{j}\right|$ that is the identity outside of $N$ extends to a self-homeomorphism of $X$ taking each $M_{j}$ to itself.

(c) For $X$ a well-ordered set, let $M_{\alpha}=\{\alpha\}$ for each $\alpha \in X$. Then all conditions in the definition of an IM complex are obviously satisfied.

(d) Suppose that $X=J \times M$, where $J$ is a well-ordered set and $M$ is a manifold. Setting $M_{\alpha}=\{\alpha\} \times M$ for each $\alpha \in J$, all conditions in the definition of an IM complex are satisfied.

(e) and (f) we leave as easy exercises.

(g) Given a locally compact IM complex $\left(X,\left\{M_{\alpha}\right\}_{\alpha \in J}\right)$, let $Y=X \cup\{\infty\}$ be the one-point compactification of $X$. Assume without loss of generality that $\infty \notin J$, and extend the order on $J$ to $K=J \cup\{\infty\}$ by declaring $\infty$ to be bigger than every member of $J$. Set $M_{\infty}=\{\infty\}$. Then $\left(Y,\left\{M_{\alpha}\right\}_{\alpha \in K}\right)$ is easily seen to be an IM complex.

(h) Let $M_{1}=(A \cup B) \backslash(A \cap B)$ and $M_{2}=A \cap B$. As usual the only thing in the definition of IM complex that requires a proof is condition (3). Given a point $p$ in $A \cap B$ let $N$ be a neighborhood of $p$ contained in a parametrized neighborhood of $p$ in $A \cap B$. If every self-homeomorphism of $A \cap B$ that is the identity outside of $N$ extends to a self-homeomorphism of $A$ and to a self-homeomorphism of $B$, then since these two homeomorphisms agree on $A \cap B$, they can be combined to yield a self-homeomorphism of $A \cup B$. The proof that the required extensions to $A$ and $B$ exist for a suitable choice of $N$ is similar to the argument used in part (a), so the details are left to the reader. 


\section{Counterexamples}

As mentioned in the introduction, Theorem 1.5 does not hold for arbitrary compact spaces. A natural place to look for counterexamples is on spaces with few selfhomeomorphisms. Spaces on which there are no self-homeomorphisms other than the identity are called rigid. The first example of a compact (Hausdorff) rigid space seems to have been given by A. S. Besicovitch [3], and additional early examples were given by J. de Groot and R. J. Wille [5]. These spaces contain homeomorphic copies of the Cantor set, and hence there are nontrivial uniform algebras defined on them by Rudin's theorem (Theorem 1.9 above). This of course establishes that Theorem 1.5] does not hold for arbitrary compact spaces, but as remarked in the introduction, it leaves open the possibility that the theorem might extend to arbitrary compact spaces under the additional hypothesis that the maximal ideal space $\mathfrak{M}_{A}$ is $X$. In addition, it is of interest to have more explicit examples. We give such examples below both with and without the additional condition that $\mathfrak{M}_{A}=X$. One of the rigid spaces of de Groot and Wille is a modification of the Swiss cheese that is familiar to researchers in uniform algebras, and our examples make use of their construction.

Example 3.1. There exists a compact rigid space $K$ and uniform algebras $A$ and $B$ on $K$ such that

(a) $\mathfrak{M}_{A}=K$ but $A \neq C(K)$, and

(b) $\mathfrak{M}_{B} \neq K$ (and hence $B \neq C(K)$ ).

Following de Groot and Wille, we begin with a closed disc $\Delta$ in the complex plane, let $\left\{a_{i}\right\}$ be a countable dense subset of the interior of $\Delta$, and choose a sequence of "propellers" in $\Delta$. The first propeller is bounded by a two-bladed curve having $a_{1}$ as its center and lying within the interior of $\Delta$. (See Figure 1.) Now proceed by induction. Suppose that the first $n-1$ propellers have been defined. Let $a_{n}^{\prime}$ be the first member of the sequence $\left\{a_{i}\right\}$ that is in no previously chosen propeller. Then choose an $(n+1)$-bladed propeller centered at $a_{n}^{\prime}$ that lies in the interior of $\Delta$ and is disjoint from all previously chosen propellers. In addition, we require the propellers to be chosen small enough that the sum of the lengths of all

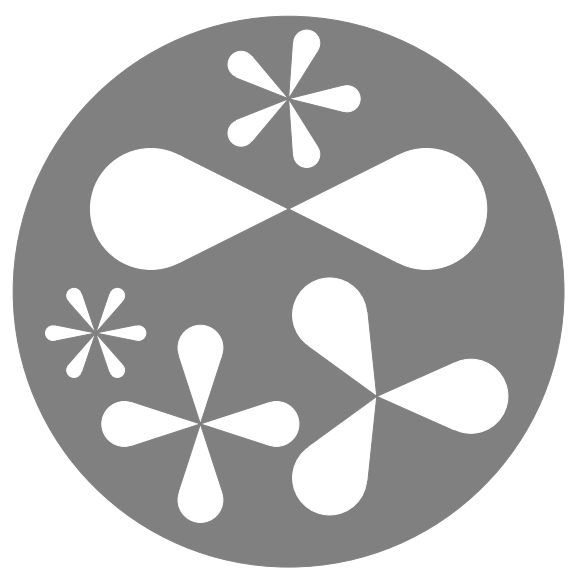

FiguRE 1 
curves bounding the propellers is finite. Let $K$ be the space obtained by removing the interiors of all the propellers from $\Delta$.

Let $\left\{a_{n}^{\prime}: n \in \mathbb{Z}_{+}\right\}$be the set of all centers of the propellers. For a sufficiently small neighborhood $U$ of $a_{n}^{\prime}$ in $K$, the set $U \backslash\left\{a_{n}^{\prime}\right\}$ has exactly $n+1$ components, each of which have $a_{n}^{\prime}$ as a limit point. For every point $p \in K \backslash\left\{a_{n}^{\prime}: n \in \mathbb{Z}_{+}\right\}$ and for a sufficiently small neighborhood $U$ of $p$ in $K$, the set $U \backslash\{p\}$ is connected. Hence every self-homeomorphism of $K$ fixes each $a_{n}^{\prime}$. Since the set $\left\{a_{n}^{\prime}: n \in \mathbb{Z}_{+}\right\}$ is dense in $K$, it follows that $K$ is rigid.

Now let $A=R(K)$, the algebra of complex-valued functions on $K$ that can be approximated uniformly on $K$ by rational functions with poles off $K$. By the usual argument used for ordinary Swiss cheeses (see for instance [6, p. 26]) we have $R(K) \neq C(K)$, and as always for planar sets $\mathfrak{M}_{R(K)}=K$.

Let $B=P(K)$, the algebra of complex-valued functions on $K$ that can be approximated uniformly on $K$ by polynomials in the complex coordinate function $z$. Then $\mathfrak{M}_{B}=\Delta$ (by [․ Theorem II.1.4]).

The definition of IM complex is reminiscent of the definition of CW complex and one may wonder whether Theorem 1.5 holds for CW complexes. In fact it does not, and we can easily construct counterexamples using the examples above.

Example 3.2. There exists a finite CW complex $X$ and uniform algebras $A$ and $B$ on $X$ that are each invariant under every self-homeomorphism of $X$ such that

(a) $\mathfrak{M}_{A}=X$ but $A \neq C(X)$, and

(b) $\mathfrak{M}_{B} \neq X$ (and hence $B \neq C(X)$ ).

Begin with a two-cell (closed disc) $Y$ containing the de Groot-Wille space $K$ above, and let $Z$ be a three-cell. Note that $K$ is a compact, connected, locally connected, metric space (see [5]), so by the Hahn-Mazurkiewicz theorem, $K$ is the continuous image of the closed unit interval. Hence there is a continuous map $g$ of the boundary of $Z$ onto $K$. Let $X$ be the adjunction space formed from $Y$ and $Z$ by means of $g$. Then $X$ is a finite CW complex, and we can regard $Y$ and the interior of $Z$ as subspaces of $X$. Now let $A=\{f \in C(X): f \mid K \in R(K)\}$ and $B=\{f \in C(X): f \mid K \in P(K)\}$. Then clearly $A \neq C(X)$ and $B \neq C(X)$. It follows from [2, Theorem 4] that $\mathfrak{M}_{A}=X$ and $\mathfrak{M}_{B} \neq X$. Clearly $A$ and $B$ are each invariant under every self-homeomorphism of $X$ that fixes each point of $K$. Thus to show the asserted invariance of $A$ and $B$ it suffices, by the rigidity of $K$, to show that each self-homeomorphism of $X$ maps $K$ onto itself. Let $h: X \rightarrow X$ be an arbitrary homeomorphism. By dimensional considerations $h(Y \backslash K) \subset Y$ and $h(\operatorname{Int}(Z)) \subset \operatorname{Int}(Z) \cup K$. Since $K$ is contained in the closures of each of $Y \backslash K$ and $\operatorname{Int}(Z)$, it follows that $h(K) \subset Y \cap[\operatorname{Int}(Z) \cup K]=K$. Since the same argument applies to $h^{-1}$, we conclude that $h(K)=K$.

Another method of constructing rigid spaces, also mentioned in [5], is to start with a line segment and successively attach differing numbers of line segments at different points. This approach can be applied to obtain examples of nontrivial uniform algebras invariant under all self-homeomorphisms of the underlying space without involving Swiss cheeses. For instance, start with the unit circle and let $\left\{a_{n}\right\}$ be a countable dense subset. For each $n$, attach $n$ line segments at $a_{n}$ in such a way as to avoid intersections and such that the lengths of the segments go to zero as $n \rightarrow \infty$. Then every self-homeomorphism of the space fixes the circle, and so the algebra of continuous functions whose restrictions to the circle lie in the disc algebra has the desired invariance. A similar example, but one defined on 
its maximal ideal space, can be obtained by replacing the circle by a disc. These examples can also be used to construct examples on CW complexes. The details are left to the reader.

Note that every finite CW complex $X$ satisfies all the conditions in the definition of an IM complex except (3) if we take $J=\{0, \ldots, \operatorname{dim} X\}$ ordered so that $\operatorname{dim} X<$ $\operatorname{dim} X-1<\ldots<1<0$ and take $M_{j}$ to be the union of all open cells of dimension $j$. Thus the CW complexes above show that condition (3) is an essential part of the definition of an IM complex; it is not a consequence of the other conditions. Note also that Theorem 2.1 gives many examples of IM complexes that are not CW complexes.

It might be tempting to imagine that if $X$ is a space such that Theorem 1.5 holds for each component of $X$, then Theorem [1.5 holds for $X$ itself, or at least that Theorem 1.5 holds for every space, each component of which is a manifold. However, this is not so. Let $S$ be a circle and let $\left\{a_{n}\right\}$ be a countable dense subset of $S$. Now let $X$ be a space consisting of $S$ together with, for each $n$, a sequence of $n$-spheres converging to $a_{n}$. More precisely, we construct $X$ as follows. For each $n \in \mathbb{Z}_{+}$, choose a sequence of $n$-spheres $S_{1}^{n}, S_{2}^{n}, \ldots$ so that all the spheres (including $S$ ) are disjoint from one another, and as a set, let $X$ be the union of all the spheres (including $S$ ). For each open set $U$ of $S$, let $\mathscr{U}=\left\{S_{j}^{n}: a_{n} \in U, j \in \mathbb{Z}_{+}\right\}$, and for each finite subset $\mathscr{F}$ of $\mathscr{U}$, let $U_{\mathscr{F}}$ be the union of $U$ and all members of $\mathscr{U}$ not in $\mathscr{F}$. Let $\mathscr{B}^{\prime}=\left\{U_{\mathscr{F}}: U\right.$ is open in $S$ and $\mathscr{F}$ is a finite subset of $\left.\mathscr{U}\right\}$. Let $\mathscr{B}^{\prime \prime}$ be the collection consisting of all the open subsets of the $S_{j}^{n}$ for all $n, j \in \mathbb{Z}_{+}$. Finally let $\mathscr{B}=\mathscr{B}^{\prime} \cup \mathscr{B}^{\prime \prime}$. Then $\mathscr{B}$ is a basis for a topology on $X$. Give $X$ the topology generated by $\mathscr{B}$. Then $X$ is a compact Hausdorff space and every component of $X$ is a sphere of some dimension. Let $A$ be the algebra of those continuous functions on $X$ whose restrictions to $S$ lie in the disc algebra. Every self-homeomorphism of $X$ fixes every point of $S$, and hence $A$ is invariant under every self-homeomorphism but clearly $A \neq C(X)$. A similar example defined on its maximal ideal space can be obtained by replacing the circle $S$ by a closed disc and taking the countable set $\left\{a_{n}\right\}$ to be dense in the disc.

\section{The Proofs of Theorems 1.5, 1.7, And 1.8}

The basic idea of the proofs of Theorems 1.5, 1.7, and 1.8 is to determine the maximal ideal space $\mathfrak{M}_{A}$ of $A$, and then to invoke an abstract uniform algebra generalization given in [11] of a well-known approximation result of Hörmander and Wermer 8]. For the reader's convenience, we quote here the result we will use.

Theorem 4.1 ([11, Theorem 1.3]). Let $A$ be a uniform algebra on a compact space $X$. Suppose that the maximal ideal space of $A$ is $X$. Suppose also that $E$ is a closed subset of $X$ such that $X \backslash E$ is an m-dimensional manifold and such that

(i) for each point $p \in X \backslash E$ there are functions $f_{1}, \ldots, f_{m} \in A$ that are $C^{1}$ on $X \backslash E$ and satisfy $d f_{1} \wedge \cdots \wedge d f_{m}(p) \neq 0$, and

(ii) the functions in $A$ that are $C^{1}$ on $X \backslash E$ separate points on $X$.

Then the essential set for $A$ is contained in $E$.

We will prove Theorem 1.8 first and then discuss the modifications needed to handle the possible presence of zero-dimensional manifolds in Theorems 1.5 and 1.7 In preparation we begin with some lemmas. 
Lemma 4.2. Let $\left(X,\left\{M_{\alpha}\right\}_{\alpha \in J}\right)$ be an IM complex with exceptional set. Given $\alpha_{0} \in J$ with $\alpha_{0}<\infty$ and distinct points $p$ and $q$ with $p \in M_{\alpha_{0}}$ and $q \in \bigcup_{\alpha \geq \alpha_{0}} M_{\alpha}$ :

(i) there is a neighborhood $W_{p}$ of $p$ in $M_{\alpha_{0}}$ such that for every point $x \in W_{p}$ there is a distinguished IM self-homeomorphism of $X$ that takes $p$ to $x$ and is the identity on $\bigcup_{\alpha>\alpha_{0}} M_{\alpha}$, and

(ii) there are disjoint neighborhoods $U_{p}$ and $U_{q}$ of $p$ and $q$ respectively in $\bigcup_{\alpha>\alpha_{0}} M_{\alpha}$ such that for every neighborhood $N_{p}$ of $p$ in $M_{\alpha_{0}}$ there is a distinguished IM self-homeomorphism of $X$ that maps $U_{p}$ into $N_{p}$ and is the identity on $U_{q}$ and on $\bigcup_{\alpha>\alpha_{0}} M_{\alpha}$.

Proof. For (i) take $W_{p}$ to be a neighborhood of $p$ in $M_{\alpha_{0}}$ as in the last condition in the definition of an IM complex with exceptional set. By shrinking $W_{p}$, we may assume that $W_{p}$ is homeomorphic to an open ball in Euclidean space and $\bar{W}_{p}$ is homeomorphic to the corresponding closed ball. Then for each point $x \in W_{p}$ there is a self-homeomorphism of $\bar{W}_{p}$ taking $p$ to $x$ and fixing $\partial W_{p}$. This homeomorphism extends to a distinguished IM self-homeomorphism of $X$.

For (ii) first note that by shrinking $W_{p}$ further, we may assume that there is a neighborhood $U_{q}$ of $q$ disjoint from $W_{p}$. Let $U_{p}$ be a neighborhood of $p$ whose closure lies in $W_{p}$. Since $W_{p}$ is homeomorphic to an open ball in Euclidean space and $\bar{W}_{p}$ to its closure, one can easily find for each neighborhood $N_{p}$ of $p$ a selfhomeomorphism of $\bar{W}_{p}$ that sends $U_{p}$ into $N_{p}$ and fixes $\partial W_{p}$. This homeomorphism extends to the desired distinguished IM self-homeomorphism of $X$.

Lemma 4.3. For $n>1$, there is a homeomorphism $\sigma:[-1,1]^{n} \rightarrow[-1,1]^{n}$ such that

(i) $\sigma$ is the identity on the boundary of $[-1,1]^{n}$ in $\mathbb{R}^{n}$,

(ii) $\sigma$ is a diffeomorphism on $(-1,1)^{n}$,

(iii) $\sigma(0)=0$,

(iv) $d \sigma_{0}^{*}\left(d x_{1}\right)=d x_{1}+d x_{n}$ and $d \sigma_{0}^{*}\left(d x_{n}\right)=d x_{n}$ (where $d \sigma_{0}^{*}$ denotes the adjoint of $\left.d \sigma_{0}\right)$.

Proof. Let $\alpha, \beta: \mathbb{R} \rightarrow \mathbb{R}$ be $C^{\infty}$ functions such that $\alpha(-1)=\alpha(1)=0, \alpha(0)=1$, $\beta(-1)=\beta(0)=\beta(1)=0, \beta^{\prime}(0)=1$, and $|\alpha(x)| \leq 1,\left|\alpha^{\prime}(x)\right| \leq 2,|\beta(x)| \leq \frac{1}{4}$ for all $x \in \mathbb{R}$. Define $\sigma:[-1,1]^{n} \rightarrow[-1,1]^{n}$ by

$$
\sigma\left(x_{1}, \ldots, x_{n}\right)=\left(x_{1}+\alpha\left(x_{1}\right) \cdots \alpha\left(x_{n-1}\right) \beta\left(x_{n}\right), x_{2}, \ldots, x_{n}\right) .
$$

Letting $\sigma_{1}$ denote the first component of $\sigma$, the conditions on $\alpha$ and $\beta$ insure that $\partial \sigma_{1} / \partial x_{1}>0$ everywhere and $\sigma_{1}\left( \pm 1, x_{2}, \ldots, x_{n}\right)= \pm 1$ for all $x_{2}, \ldots, x_{n}$; it follows that $\sigma$ is a well-defined homeomorphism. Conditions (i) and (iii) are obvious. The strict positivity of $\partial \sigma_{1} / \partial x_{1}$ gives invertibility of the derivative $D \sigma$ of $\sigma$, so (ii) follows from the inverse function theorem. The matrix of $D \sigma_{0}$ is the elementary matrix with a 1 in the upper right corner, and this implies (iv).

Lemma 4.4. Let $K$ be a topological space and $p$ be a point of $K$ that has a neighborhood $N$ that is a smooth manifold. Then there is a neighborhood $N_{1}$ of $p$ such that for every pair of linearly independent vectors $v$ and $w$ in the (real) cotangent space $T_{p}^{*} N$ of $N$ at $p$ there is a self-homeomorphism $h$ of $X$ that is the identity outside of $N_{1}$, is smooth on $N_{1}$, and satisfies $d h^{*}(v)=v+w$ and $d h^{*}(w)=w$ (where $d h^{*}$ denotes the adjoint of $d h$ ). 
Proof. Let $\psi:[-1,1]^{n} \rightarrow X$ be a map that extends to a parametrization of a neighborhood of $p$ in $N$ and satisfies $\psi(0)=p$. By making a linear change of coordinates in $\mathbb{R}^{n}$, we may assume that $d \psi_{0}{ }^{*}(v)=d x_{1}$ and $d \psi_{0}{ }^{*}(w)=d x_{n}$. Using $\psi$ we can transfer the self-homeomorphism $\sigma$ in Lemma4.3 to a self-homeomorphism of $\psi\left([-1,1]^{n}\right)$ that can be extended to a self-homeomorphism $h$ of $X$ by making it the identity outside of $\psi\left([-1,1]^{n}\right)$. A trivial computation shows that $d h^{*}(v)=v+w$ and $d h^{*}(w)=w$.

Lemma 4.5. Let $K$ be a compact space, and let $p$ be a point of $K$. Suppose that there is a homeomorphism $\varphi$ of some neighborhood $N$ of $p$ with $\mathbb{R}^{n}$ and regard $N$ as a smooth manifold by taking the single coordinate system $(N, \varphi)$ as an atlas. Let $A$ be a uniform algebra on $K$ and assume that $A$ is invariant under every selfhomeomorphism of $K$ that is the identity outside of $N$. Then there is a neighborhood $U$ of $p$ contained in $N$ such that

(i) there are functions $f_{1}, \ldots, f_{n}$ in $A$ that are smooth on $U$ and such that $d f_{1} \wedge \cdots \wedge d f_{n}(p) \neq 0$, and

(ii) the functions in $A$ that are smooth on $U$ are dense in $A$.

Proof. Without loss of generality we may assume that $\varphi(p)=0$, and letting $B$ denote the open unit ball in $\mathbb{R}^{n}$, that $\varphi^{-1}(B)$ is contained in the neighborhood $N_{1}$ given by Lemma 4.4. Let $U=\varphi^{-1}(B)$. Then $\bar{U}=\varphi^{-1}(\bar{B})$.

We first establish (ii) by a convolution argument. The map $x \mapsto x / \sqrt{1-\|x\|^{2}}$ takes $B$ diffeomorphically onto $\mathbb{R}^{n}$. (The inverse map is given by $y \mapsto y / \sqrt{1+\|y\|^{2}}$.) We can thus use the map to transfer any self-homeomorphism of $\mathbb{R}^{n}$ to a selfhomeomorphism of $B$. For each $y \in \mathbb{R}^{n}$, let $h_{y}$ be the self-homeomorphism of $B$ obtained in this way from the translation $x \mapsto x-y$ on $\mathbb{R}^{n}$. The reader can verify that $h_{y}$ extends to a self-homeomorphism of $\bar{B}$ that is the identity on $\partial B$. Then $h_{y}$ induces a self-homeomorphism $\tilde{h}_{y}$ of $\bar{U}$ making the following diagram commute:

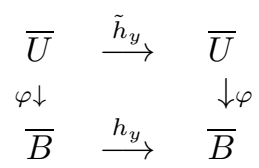

Finally define a map $H_{y}: K \rightarrow K$ by

$$
H_{y}(x)= \begin{cases}\tilde{h}_{y}(x), & x \in \bar{U}, \\ x, & x \in K \backslash U .\end{cases}
$$

Note that $H_{y}$ is well defined since $\tilde{h}_{y}$ is the identity on $\partial U$, and $H_{y}$ is a selfhomeomorphism of $K$ because both $\bar{U}$ and $K \backslash U$ are closed in $K$. The reader can verify that the map $(x, y) \mapsto H_{y}(x)$ is continuous on $K \times \mathbb{R}^{n}$. The reader can also verify that

$$
\left\|h_{y}(w)-w\right\|<\|y\| \quad \text { for all } w \in B \text { and } y \in \mathbb{R}^{n} .
$$

Now given $f \in A$ and $\varepsilon>0$ we construct a function $f_{\varepsilon} \in A$ with $\left\|f_{\varepsilon}-f\right\|_{\infty}<\varepsilon$ such that $f_{\varepsilon}$ is smooth on $U$. (Here $\|\cdot\|_{\infty}$ denotes the supremum norm.) Choose a nonnegative smooth function $g$ with support in $\left\{y \in \mathbb{R}^{n}:\|y\|<\delta\right\}$ such that $\int_{\mathbb{R}^{n}} g(y) d y=1$ (where $\delta>0$ is to be specified below). Define $f_{\varepsilon}$ on $K$ by

$$
f_{\varepsilon}(x)=\int_{\mathbb{R}^{n}} f\left(H_{y}(x)\right) g(y) d y .
$$


Since for each $x$ the integrand is a compactly supported continuous function of $y$, there is no issue of existence of the integral. Note that $f$ induces, via the homeomorphisms $\varphi$ and $x \mapsto x / \sqrt{1-\|x\|^{2}}$, a function $\tilde{f}$ on $\mathbb{R}^{n}$ making the following diagram commute for each $y \in \mathbb{R}^{n}$ :

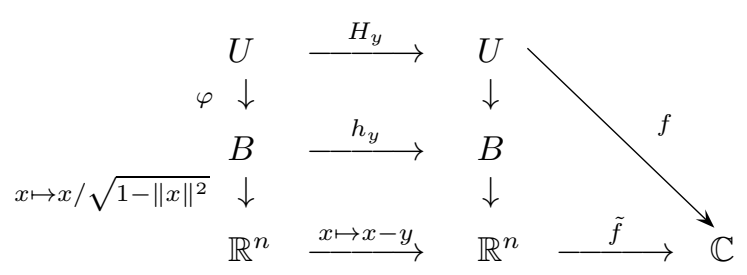

If we let $z=\varphi(x) / \sqrt{1-\|\varphi(x)\|^{2}}$ denote the point in $\mathbb{R}^{n}$ corresponding to the point $x$ in $U$, then

$$
\tilde{f}(z-y)=f\left(H_{y}(x)\right) \quad \text { for all } y \in \mathbb{R}^{n},
$$

so

$$
f_{\varepsilon}(x)=\int_{\mathbb{R}^{n}} \tilde{f}(z-y) g(y) d y=(\tilde{f} * g)(z) .
$$

Thus $f_{\varepsilon}$ is smooth on $U$.

By the uniform continuity of $f \circ \varphi^{-1}$ on the compact space $\bar{B}$, there is a $\delta>0$ such that

$$
\left|\left(f \circ \varphi^{-1}\right)\left(t_{1}\right)-\left(f \circ \varphi^{-1}\right)\left(t_{2}\right)\right|<\varepsilon \quad \text { whenever }\left\|t_{1}-t_{2}\right\|<\delta .
$$

We claim that

$$
\left|\left(f \circ H_{y}\right)(x)-f(x)\right|<\varepsilon \quad \text { for all } x \in K \text { and }\|y\|<\delta .
$$

This is obvious for $x$ in $K \backslash U$, while for $x$ in $U$ we have

$$
\begin{aligned}
\left|\left(f \circ H_{y}\right)(x)-f(x)\right| & =\left|\left(f \circ \tilde{h}_{y}\right)(x)-f(x)\right| \\
& =\left|\left(f \circ \varphi^{-1}\right)\left(h_{y}(\varphi(x))\right)-\left(f \circ \varphi^{-1}\right)(\varphi(x))\right| \\
& <\varepsilon \quad(\text { by }(1) \text { and }(2)) .
\end{aligned}
$$

Since the support of $g$ lies in $\left\{y \in \mathbb{R}^{n}:\|y\|<\delta\right\}$, inequality (3) gives that for all $x$ in $K$

$$
\begin{aligned}
\left|f_{\varepsilon}(x)-f(x)\right| & =\left|\int\left[f\left(H_{y}(x)\right)-f(x)\right] g(y) d y\right| \\
& \leq \int\left|f\left(H_{y}(x)\right)-f(x)\right| g(y) d y \\
& <\varepsilon
\end{aligned}
$$

so $\left\|f_{\varepsilon}-f\right\|_{\infty}<\varepsilon$.

The continuity of the map $(x, y) \mapsto H_{y}(x)$ together with the compactness of $K$ implies that for each $y \in \mathbb{R}^{n}$ and $\eta>0$ there is a neighborhood $V$ of $y$ such that for all $y^{\prime} \in V$ and for all $x \in K$ we have

$$
\left|f\left(H_{y}(x)\right) g(y)-f\left(H_{y^{\prime}}(x)\right) g\left(y^{\prime}\right)\right|<\eta .
$$

It follows that $f_{\varepsilon}$ can be uniformly approximated by a Riemann sum $\sum c_{j}\left(f \circ H_{y_{j}}\right)$. Since the invariance hypothesis on $A$ implies the function $f \circ H_{y}$ is in $A$ for every $y$, we conclude that $f_{\varepsilon}$ is in $A$. 
We now turn to establishing (i). Let $V$ be the vector subspace of the complexified cotangent space to $N$ at $p$ consisting of the differentials (at $p$ ) of functions in the algebra $A$. Note that (i) is equivalent to the statement that $V$ is the full complexified cotangent space. By (ii) there must be a function $a$ in $A$ that is smooth on $U$ and whose derivative does not vanish identically on $U$. Let $q$ be a point of $U$ such that $d a(q) \neq 0$. Set

$$
y=\left[\varphi(p) / \sqrt{1-\|\varphi(p)\|^{2}}\right]-\left[\varphi(q) / \sqrt{1-\|\varphi(q)\|^{2}}\right]
$$

and $f=a \circ H_{y}$. Then $f$ is in $A$ and $H_{y}(p)=q$, so $d f(p)=d a(q) \circ d H_{y}(p) \neq 0$. Write $d f(p)=v+i w$ with $v$ and $w$ in the real cotangent space.

We claim there is some nonzero real cotangent vector in $V$. If $v$ and $w$ are linearly dependent, then $v=\alpha u$ and $w=\beta u$ for some $\alpha, \beta \in \mathbb{R}$ and real cotangent vector $u$, whence $d f(p)=(\alpha+i \beta) u$, so $u$ is in $V$. If $v$ and $w$ are linearly independent, then recalling that $U$ is contained in the neighborhood $N_{1}$ given by Lemma 4.4, there is a self-homeomorphism $h$ of $K$ that is the identity outside of $N$ and smooth on $U$ satisfying $d h^{*}(v)=v+w$ and $d h^{*}(w)=w$. Then

$$
d(f \circ h)=d h^{*}(d f)=d h^{*}(v+i w)=(v+w)+i w .
$$

Since $f \circ h$ is in $A$, we get that the real cotangent vector $w=[(v+w)+i w]-[v+i w]$ is in $V$.

Now let $g$ be a function in $A$ such that $d g(p)$ is a real cotangent vector, let $v_{1}=d g(p)$, and choose real cotangent vectors $v_{2}, \ldots, v_{n}$ such that $\left\{v_{1}, \ldots, v_{n}\right\}$ is a basis for the real cotangent space $T_{p}^{*} N$. Again applying Lemma 4.4, there are self-homeomorphisms $\sigma_{2}, \ldots, \sigma_{n}$ such that for each $j=2, \ldots, n$, the function $g \circ \sigma_{j}$ is in $A$ and $d \sigma_{j}^{*}\left(v_{1}\right)=v_{1}+v_{j}$. Then

$$
d\left(g \circ \sigma_{j}\right)=d \sigma_{j}^{*}(d g)=d \sigma_{j}^{*}\left(v_{1}\right)=v_{1}+v_{j} .
$$

It follows that $V$ equals the complexified cotangent space to $N$ at $p$.

We now prove Theorem 1.8 .

Proof of Theorem 1.8. Let $\left\{M_{\alpha}\right\}_{\alpha \in J}$ be as in the definition of an IM complex with exceptional set, and let $C=M_{\infty}$. We divide the proof into steps.

Step 1. We show that $\mathfrak{M}_{A}=X \cup \mathfrak{M}_{\overline{A \mid C}}$. (Here $\overline{A \mid C}$ denotes the uniform algebra on $C$ obtained by taking the uniform closure of the set of restrictions of functions in $A$ to $C$, and of course we identify $\mathfrak{M}_{\overline{A \mid C}}$ with a subset of $\mathfrak{M}_{A}$ in the obvious way. In the case where $C=\emptyset$ we get $\mathfrak{M}_{A}=X$.)

Let $\varphi \in \mathfrak{M}_{A} \backslash X$ be arbitrary. We will show that $\varphi$ is in $\mathfrak{M}_{\overline{A \mid C}}$ by showing that there is a representing measure for $\varphi$ supported on $C$. This we establish by applying transfinite induction to show that for every $\alpha_{0} \in J$ there is a representing measure for $\varphi$ supported on $\bigcup_{\alpha \geq \alpha_{0}} M_{\alpha}$. For notational convenience we will denote $\bigcup_{\alpha \geq \beta} M_{\alpha}$ by $K_{\beta}$.

Let $\alpha_{0} \in J$ be arbitrary and suppose as the induction hypothesis that for all $\alpha<\alpha_{0}$ there is a representing measure for $\varphi$ supported on $K_{\alpha}$. We must show there is a representing measure for $\varphi$ supported on $K_{\alpha_{0}}$. If $\alpha_{0}$ is the smallest member of $J$, then $K_{\alpha_{0}}=X$, so no argument is needed. For the other values of $\alpha_{0}$ the proof splits into two cases depending on whether or not $\alpha_{0}$ has an immediate predecessor. 
Case 1. The index $\alpha_{0}$ has an immediate predecessor $\gamma$.

Let $A_{\gamma}$ denote the uniform algebra $\overline{A \mid K_{\gamma}}$. By the induction hypothesis there is a representing measure $\mu$ for $\varphi$ supported on $K_{\gamma}$. It follows that $\varphi$ induces a multiplicative linear functional $\tilde{\varphi}$ on $A_{\gamma}$ such that $\tilde{\varphi}\left(f \mid K_{\gamma}\right)=\varphi(f)$ for each function $f$ in $A$. Now let $\sigma$ be a Jensen measure for $\tilde{\varphi}$ supported on the Šilov boundary of $A_{\gamma}$. Then $\sigma$ is also a Jensen measure for $\varphi$. We will show that in fact $\sigma$ is supported on $K_{\gamma} \backslash M_{\gamma}=K_{\alpha_{0}}$.

Assume to get a contradiction that the support of $\sigma$ intersects $M_{\gamma}$ and let $p$ be a point in the intersection. Since $\varphi \notin X$, the measure $\sigma$ is not a point mass, so there is some other point $q$ in the support of $\sigma$.

If $h$ is a distinguished IM self-homeomorphism as in the statement of the theorem, then $h$ sends $K_{\gamma}$ into itself and $A_{\gamma}$ is invariant under $h$; more precisely the map $C\left(K_{\gamma}\right) \rightarrow C\left(K_{\gamma}\right)$ given by $f \mapsto f \circ\left(h \mid K_{\gamma}\right)$ sends $A_{\gamma}$ into itself. The set of generalized peak points for a uniform algebra is always dense in the Šilov boundary (this follows from [6. Theorem II.12.10] for instance), so every neighborhood of $p$ in $K_{\gamma}$, and hence in $M_{\gamma}$, contains a generalized peak point for $A_{\gamma}$. Since $M_{\gamma}$ is a manifold, we in fact get a genuine peak point. Now by Lemma 4.2(i) there is a distinguished IM self-homeomorphism of $X$ under which $A_{\gamma}$ is invariant and that takes $p$ to a peak point for $A_{\gamma}$. It follows that $p$ is itself a peak point for $A_{\gamma}$.

Applying Lemma 4.2(ii), we get disjoint neighborhoods $U_{p}$ and $U_{q}$ of $p$ and $q$ respectively in $K_{\gamma}$ such that for every neighborhood $N_{p}$ of $p$ in $M_{\alpha_{0}}$ there is a distinguished IM self-homeomorphism of $X$ that maps $U_{p}$ into $N_{p}$ and is the identity on $U_{q}$. Since $p$ and $q$ are in supp $\sigma$, every neighborhood of each of these points has positive $\sigma$ measure. In particular $\sigma\left(U_{p}\right)>0$ and $\sigma\left(U_{q}\right)>0$. Consequently, there is an $\varepsilon>0$ small enough that

$$
(\log \varepsilon) \sigma\left(U_{q}\right)<\log (1 / 2) \text { and }(\log \varepsilon) \sigma\left(U_{p}\right)+\log 2<\log (1 / 2) .
$$

Let $f$ be a function in $A_{\gamma}$ that peaks at $p$. By replacing $f$ by a sufficiently high power of itself, we may assume that $|f|<\varepsilon$ on $U_{q}$. Now let $N_{p}$ be a neighborhood of $p$ on which $|1-f|<\varepsilon$. Choose a distinguished IM self-homeomorphism of $X$ as in Lemma 4.2(ii). Let $h$ denote the restriction of this homeomorphism to $K_{\gamma}$ and consider the function $f \circ h$. By the invariance of $A_{\gamma}$, we have $f \circ h \in A_{\gamma}$. Note that $|f \circ h|<\varepsilon$ on $U_{q}$ and $|1-(f \circ h)|<\varepsilon$ on $U_{p}$. Also $|f \circ h| \leq 1$ everywhere. Since $\sigma$ is a Jensen measure for $\tilde{\varphi}$, this gives

$$
\log |\tilde{\varphi}(f \circ h)| \leq \int_{K_{\gamma}} \log |f \circ h| d \sigma \leq \int_{U_{q}} \log |f \circ h| d \sigma \leq(\log \varepsilon) \sigma\left(U_{q}\right)
$$

and

$$
\begin{aligned}
\log |1-\tilde{\varphi}(f \circ h)| & =\log |\tilde{\varphi}(1-(f \circ h))| \\
& \leq \int_{K_{\gamma}} \log |1-(f \circ h)| d \sigma \\
& =\int_{U_{p}} \log |1-(f \circ h)| d \sigma+\int_{K_{\gamma} \backslash U_{p}} \log |1-(f \circ h)| d \sigma \\
& \leq(\log \varepsilon) \sigma\left(U_{p}\right)+\log 2 .
\end{aligned}
$$

So $\log |\tilde{\varphi}(f \circ h)|<\log (1 / 2)$ and $\log |1-\tilde{\varphi}(f \circ h)|<\log (1 / 2)$ by the inequalities (4). Thus $|\tilde{\varphi}(f \circ h)|<1 / 2$ and $|1-\tilde{\varphi}(f \circ h)|<1 / 2$, a contradiction. This contradiction shows that $\sigma$ is supported on $K_{\alpha_{0}}$. 
Case 2. The index $\alpha_{0}$ has no immediate predecessor.

By the induction hypothesis for each $\gamma<\alpha_{0}$ there is a representing measure $\mu_{\gamma}$ for $\varphi$ supported on $K_{\gamma}$. Given $f \in A \mid K_{\alpha_{0}}$, let $\tilde{f}$ be an extension of $f$ to a function in $A$ and define $\tilde{\varphi}(f)=\varphi(\tilde{f})$. We claim that the value of $\tilde{\varphi}(f)$ is independent of the choice of extension $\tilde{f}$. First note that since each $\mu_{\gamma}$ represents $\varphi$, we have $\tilde{\varphi}(f)=\int \tilde{f} d \mu_{\gamma}$. Now suppose $\tilde{f}_{1}$ and $\tilde{f}_{2}$ are two different extensions of $f$. Let $\varepsilon>0$ be arbitrary and choose an open set $U$ of $X$ containing $K_{\alpha_{0}}$ on which $\sup _{x \in U}\left|\tilde{f}_{1}(x)-\tilde{f}_{2}(x)\right|<\varepsilon$. Since $K_{\alpha_{0}}=\bigcap_{\gamma<\alpha_{0}} K_{\gamma}$, a simple compactness argument shows that $U \supset K_{\gamma_{0}}$ for some $\gamma_{0}<\alpha_{0}$. Thus

$$
\begin{aligned}
\left|\varphi\left(\tilde{f}_{1}\right)-\varphi\left(\tilde{f}_{2}\right)\right| & =\left|\int \tilde{f}_{1} d \mu_{\gamma_{0}}-\int \tilde{f}_{2} d \mu_{\gamma_{0}}\right| \\
& =\left|\int\left(\tilde{f}_{1}-\tilde{f}_{2}\right) d \mu_{\gamma_{0}}\right| \\
& \leq \int\left|\tilde{f}_{1}-\tilde{f}_{2}\right| d \mu_{\gamma_{0}}<\varepsilon .
\end{aligned}
$$

Since $\varepsilon>0$ was arbitrary this shows that $\tilde{\varphi}(f)$ is independent of the extension $\tilde{f}$. Thus we have a well-defined functional $\tilde{\varphi}$ defined on $A \mid K_{\alpha_{0}}$. It is clear that $\tilde{\varphi}$ is linear and multiplicative. Also, an argument similar to the one above shows that $\tilde{\varphi}$ is norm-decreasing, and hence $\tilde{\varphi}$ extends to a multiplicative linear functional on $A_{\alpha_{0}}=\overline{A \mid K}_{\alpha_{0}}$. Let $\sigma$ be a representing measure for $\tilde{\varphi}$. Then $\sigma$ is also a representing measure for $\varphi$ supported on $K_{\alpha_{0}}$.

This concludes Step 1.

Step 2. We show that $X \cap \mathfrak{M}_{\overline{A \mid C}}=C$.

Since the inclusion $X \cap \mathfrak{M}_{\overline{A \mid C}} \supset C$ is obvious, it suffices to show that no point of $X \backslash C$ lies in $\mathfrak{M}_{\overline{A \mid C}}$. So let $x_{0}$ in $X \backslash C$ be arbitrary, let $\alpha_{0}$ be the index such that $x_{0}$ is in $M_{\alpha_{0}}$, and let $N$ be a neighborhood of $x_{0}$ in $M_{\alpha_{0}}$ as in the last condition in the definition of IM complex with exceptional set. Then for some point $x_{1}$ in $N$ distinct from $x_{0}$ there is a self-homeomorphism $h$ of $X$ that sends $x_{0}$ to $x_{1}$ and is the identity outside of $N$. In particular $h$ is the identity on $C$. Now choose a function $f$ in $A$ such that $f\left(x_{0}\right) \neq f\left(x_{1}\right)$. Then $f$ and $f \circ h$ are functions in $A$ that agree on $C$ but differ at $x_{0}$. Hence $x_{0}$ cannot belong to $\mathfrak{M}_{\overline{A \mid C}}$.

Step 3. We complete the proof by showing that for every $\alpha \in J$, the essential set for $A$ is contained in $\bigcup_{\beta>\alpha} M_{\beta}$, and hence in particular the essential set for $A$ is contained in $M_{\infty}$, as desired.

Let $\alpha_{0} \in J$ be arbitrary and assume as the induction hypothesis that the essential set for $A$ is contained in $\bigcup_{\beta>\alpha} M_{\beta}$ for every $\alpha<\alpha_{0}$. If $\alpha_{0}$ has no immediate predecessor, we get at once that the essential set for $A$ is contained in $\bigcup_{\beta \geq \alpha_{0}} M_{\beta}$. If $\alpha_{0}$ has an immediate predecessor $\gamma$, we get that the essential set for $A$ is contained in $\bigcup_{\beta \geq \gamma} M_{\beta}$. For notational convenience let $K=\bigcup_{\beta \geq \gamma} M_{\beta}$. It is easy to show that the restriction of a uniform algebra to a set containing the essential set is always closed. By Step 1, $\mathfrak{M}_{A \mid K} \subset \mathfrak{M}_{A}=X \cup \mathfrak{M}_{\overline{A \mid C}}$. It is easily shown that no point of $X \backslash K$ lies in $\mathfrak{M}_{A \mid K}$. Since $\mathfrak{M}_{A \mid K} \supset \mathfrak{M}_{\overline{A \mid C}}$, we conclude that $\mathfrak{M}_{A \mid K}=K \cup \mathfrak{M}_{\overline{A \mid C}}$. Furthermore, by Step $2 K \cap \mathfrak{M}_{\overline{A \mid C}}=C$. Hence $K \backslash C=\mathfrak{M}_{A \mid K} \backslash \mathfrak{M}_{\overline{A \mid C}}$, so $K \backslash C$ is open in $\mathfrak{M}_{A \mid K}$. Consequently, $M_{\gamma}$ is open in $\mathfrak{M}_{A \mid K}$. 
Now let $p \in M_{\gamma}$ be arbitrary. Let $N$ be a neighborhood of $p$ in $M_{\gamma}$ as in the last condition in the definition of an IM complex with exceptional set. By shrinking $N$, we may assume that $N$ is homeomorphic to $\mathbb{R}^{m}$. Let $\varphi$ be a homeomorphism of $N$ with $\mathbb{R}^{m}$ and regard $N$ as a smooth manifold by taking the single coordinate system $(N, \varphi)$ as an atlas. By Lemma 4.5 there is a neighborhood $U$ of $p$ contained in $N$ such that

(i) there are functions $f_{1}, \ldots, f_{m}$ in $A \mid K$ that are smooth on $U$ and such that $d f_{1} \wedge \cdots \wedge d f_{m}(p) \neq 0$, and

(ii) the functions in $A \mid K$ that are smooth on $U$ separate points on $K$.

Shrinking $U$ we may assume that $d f_{1} \wedge \cdots \wedge d f_{m}(q) \neq 0$ for all $q \in U$. Now applying [11, Theorem 1.3] (quoted above as Theorem 4.1) to $A \mid K$ (with $E=K \backslash U$ ), we conclude that the essential set for $A \mid K$ is contained in $K \backslash U$. It follows that the essential set for $A$ is also contained in $K \backslash U$. In particular $p$ is not in the essential set for $A$. Since $p \in M_{\gamma}$ was arbitrary, we conclude that the essential set for $A$ is contained in $K \backslash M_{\gamma}=\bigcup_{\alpha \geq \alpha_{0}} M_{\alpha}$. This completes the induction and the proof.

We now turn to the proof of Theorems 1.5 and 1.7. Since the proof is similar to the proof of Theorem 1.8, we merely indicate the modifications needed.

Proof of Theorems 1.5] and 1.7. Note that Theorem 1.5] is subsumed by Theorem 1.7. Step 1 of the above proof goes through as before but simplifies somewhat since we now have $C=\emptyset$ and the conclusion is $\mathfrak{M}_{A}=X$. Because $C=\emptyset$, Step 2 is no longer needed. Step 3 goes through as before except that we must allow for the possibility that $M_{\gamma}$ is a zero-dimensional manifold. To handle that case we need a zero-dimensional form of [11, Theorem 1.3]. Fortunately the needed result is an immediate consequence of the Silov idempotent theorem as we next observe.

Proposition 4.6. Let $A$ be a uniform algebra on $X$. Suppose that $\mathfrak{M}_{A}=X$ and $E$ is a closed subset of $X$ such that $X \backslash E$ is a discrete space. Then $A=\{g \in C(X)$ : $g|E \in A| E\}$.

Proof. Let $p \in X \backslash E$ be arbitrary. The set $\{p\}$ is both open and closed in $X$, so by the Silov idempotent theorem [6, Corollary III.6.5] the function that is identically zero on $X \backslash\{p\}$ and 1 at $p$ is in $A$. Since every function on $X$ that is identically zero on $X \backslash\{p\}$ is a scalar multiple of this function, we conclude that $p$ lies outside the essential set for $A$. The conclusion follows at once.

Step 2 in the proof of Theorem 1.8 breaks down when zero-dimensional manifolds are allowed, and in fact, Theorem 1.8 becomes false in this case. For a simple example, let $S=\{z \in \mathbb{C}:|z|=1\}$ be the unit circle in the plane, let $X=$ $S \cup\left\{1-\frac{1}{n}: n \in \mathbb{Z}_{+}\right\}$, let $J=\mathbb{Z}_{+} \cup\{\infty\}$, for each $n \in \mathbb{Z}_{+}$let $M_{n}=\left\{1-\frac{1}{n}\right\}$, and let $M_{\infty}=S$. Then $X$ is an IM complex with exceptional set $M_{\infty}$ (and can also be regarded as an IM complex without an exceptional set). Now let $A$ be the restriction of the disc algebra to $X$. The identity is the only distinguished IM self-homeomorphism of $X$ that is the identity on $M_{\infty}$, so $A$ has the required invariance. Nevertheless, the essential set for $A$ is not contained in $M_{\infty}$. In fact $A$ is antisymmetric, so the essential set of $A$ is all of $X$. Note that $\mathfrak{M}_{A \mid M_{\infty}}$ is the closed unit disc, and thus the conclusion of Step 2 in the proof of Theorem 1.8 does not hold. 


\section{ZERO-DIMENSIONAL IM COMPLEXES AND SPACES ON WHICH THERE ARE NO NONTRIVIAL UNIFORM ALGEBRAS}

By a zero-dimensional IM complex we mean an IM complex in which each manifold is zero-dimensional. Note that such a space can always be expressed as an IM complex in which each manifold is a singleton set. As mentioned in the introduction, it turns out that these spaces are exactly the scattered spaces.

Theorem 5.1. A space $X$ is a zero-dimensional IM complex if and only if $X$ is scattered.

Since obviously the only distinguished IM self-homeomorphism on an IM complex $\left(X,\left\{M_{\alpha}\right\}\right)$ with each $M_{\alpha}$ a singleton is the identity map, we get as an immediate consequence that Theorem 1.7 contains the theorem of Rudin (quoted in the introduction as Theorem 1.10) that there are no nontrivial uniform algebras on scattered spaces.

Theorem 5.1 is an immediate consequence of the following result. (Given a wellordered set $X$, by a section of $X$ we mean a subset $J$ of $X$ with the property that if $a \in J$ and $b<a$, then $b \in J$. Equivalently the sections of $X$ are $X$ itself and the sets of the form $\{x \in X: x<\alpha\}$ for $\alpha \in J$.)

Theorem 5.2. A (not necessarily Hausdorff) space $X$ is scattered if and only if there is a well-ordering on $X$ such that the topology on $X$ is finer than the topology in which the open sets are the sections.

Proof. The "if" direction is trivial; if a well-ordering as in the statement of the theorem exists and $A$ is an arbitrary subset of $X$, then the smallest element of $A$ is an isolated point of $A$, and hence $X$ is scattered. For the proof of the converse, suppose $X$ is scattered and let $K$ be a well-ordered set of cardinality strictly greater than $X$. We will prove the following assertion that is clearly equivalent to the desired conclusion: there exists a bijection from a section $\tilde{K}$ of $K$ onto $X$ under which the image of each section of $\tilde{K}$ is open in $X$. Let $\mathscr{P}$ be the collection of all ordered pairs $\left(J^{\prime}, f^{\prime}\right)$ such that $J^{\prime}$ is a section of $K$ and $f^{\prime}$ is an injective function from $J^{\prime}$ into $X$ such that the image of each section of $J^{\prime}$ under $f^{\prime}$ is open in $X$. Partially order $\mathscr{P}$ by declaring $\left(J^{\prime}, f^{\prime}\right) \leq\left(J^{\prime \prime}, f^{\prime \prime}\right)$ to mean that $J^{\prime} \subset J^{\prime \prime}$ and $f^{\prime \prime}=f^{\prime}$ on $J^{\prime}$. It is easily shown that every chain in $\mathscr{P}$ has an upper bound in $\mathscr{P}$. Therefore, by Zorn's lemma, $\mathscr{P}$ has a maximal element $(J, f)$. Since the cardinality of $K$ is strictly greater than that of $X$, necessarily $J$ is a proper subset of $K$. Let $\gamma$ be the smallest element of $K \backslash J$. If $f$ is not surjective, then $X \backslash f(J)$ has an isolated point $p$, and we can extend $f$ to $J \cup\{\gamma\}$ by sending $\gamma$ to $p$, contradicting the maximality of $(J, f)$. Hence $f$ is surjective, and the proof is complete.

As a simple example of a space that is not an IM complex but for which the conclusion of Theorem 1.5 still holds, we conclude by showing that there are no nontrivial uniform algebras invariant under every self-homeomorphism on the Cantor set. This provides an interesting, though unsurprising, contrast with the situation where no invariance is assumed, since as noted in the introduction, by theorems of Rudin, there is a nontrivial uniform algebra on a metric space if and only if the space contains a Cantor set.

Theorem 5.3. If $A$ is a uniform algebra on the Cantor set $X$ that is invariant under every self-homeomorphism of $X$, then $A=C(X)$. 
The proof uses the following lemma.

Lemma 5.4. Let $X$ be a space with the following two properties:

(i) for every point $p$ of $X$ there is a neighborhood $W_{p}$ of $p$ such that for every point $x \in W_{p}$ there is a self-homeomorphism of $X$ mapping $p$ to $x$, and

(ii) for every pair of distinct points $p$ and $q$ in $X$ there exist disjoint neighborhoods $U_{p}$ and $U_{q}$ of $p$ and $q$ respectively, such that for every neighborhood $N_{p}$ of $p$ there is a self-homeomorphism of $X$ that maps $U_{p}$ into $N_{p}$ and is the identity on $U_{q}$.

Then every uniform algebra $A$ on $X$ that is invariant under every self-homeomorphism of $X$ has maximal ideal space $X$.

Proof. The basic idea of the proof is the same as that used in Case 1 of Step 1 in the proof of Theorem 1.8. Assume to get a contradiction that there is a point $\varphi \in \mathfrak{M}_{A} \backslash X$. Let $\sigma$ be a Jensen measure for $\varphi$ supported on the Šilov boundary for $A$. Since $\varphi \notin X$, the measure $\sigma$ is not a point mass, so the support of $\sigma$ contains at least two distinct points $p$ and $q$. Since the set of generalized peak points is always dense in the Šilov boundary, every neighborhood of $p$ contains a generalized peak point. Hence by condition (i) there is a self-homeomorphism of $X$ that sends $p$ to a generalized peak point. Since $A$ is invariant under this self-homeomorphism, it follows that $p$ itself is a generalized peak point. Let $U_{p}$ and $U_{q}$ be as in condition (ii). Since $p$ and $q$ are each in the support of $\sigma$, we have $\sigma\left(U_{p}\right)>0$ and $\sigma\left(U_{q}\right)>0$. Consequently, there is an $\varepsilon>0$ small enough that $(\log \varepsilon) \sigma\left(U_{q}\right)<\log (1 / 2)$ and $(\log \varepsilon) \sigma\left(U_{p}\right)+\log 2<\log (1 / 2)$. Since $p$ is a generalized peak point, there is a function $f$ in $A$ of supremum norm 1 such that $f(p)=1$ and $|f(x)|<1$ for all $x \in X \backslash U_{p}$. By replacing $f$ by a sufficiently high power of itself, we can assume that $|f|<\varepsilon$ on $X \backslash U_{p}$. Now let $N_{p}$ be a neighborhood of $p$ on which $|1-f|<\varepsilon$. Using condition (ii), choose a self-homeomorphism $h$ of $X$ that maps $U_{p}$ into $N_{p}$ and is the identity on $U_{q}$. By the invariance of $A$, the function $f \circ h$ is in $A$. Note that $|f \circ h|<\varepsilon$ on $U_{q}$ and $|1-(f \circ h)|<\varepsilon$ on $U_{p}$, and the supremum norm of $f \circ h$ is 1. Now an exact repetition of the computations done at the end of Case 1 of Step 1 in the proof of Theorem 1.8 shows that $|\varphi(f \circ h)|<1 / 2$ and $|1-\varphi(f \circ h)|<1 / 2$, our desired contradiction.

Proof of Theorem 5.3. A corollary of the Šilov idempotent theorem [1, p. 48, Corollary 2] asserts that if the maximal ideal space of a uniform algebra is totally disconnected, then $A$ contains all continuous functions. Thus it suffices to show that $A$ satisfies conditions (i) and (ii) of the above lemma.

We view the Cantor set as the product space $\{0,1\}^{\omega}$. Since this is a topological group (under pointwise addition, modulo 2), the self-homeomorphisms of $X$ act transitively, and so condition (i) holds. As to condition (ii), first note that by the transitivity of the self-homeomorphism group of $X$, we may assume without loss of generality that $p$ is the zero sequence. Then since the map that interchanges two terms in a sequence is a self-homeomorphism of $\{0,1\}^{\omega}$, we may assume without loss of generality that the first entry of $q$ is a one. Now let $X_{j_{1} \cdots j_{k}}$ denote the set of sequences of 0 's and 1's that begin with $j_{1}, \ldots, j_{k}$, that is, set $X_{j_{1} \cdots j_{k}}=\left\{\left(x_{n}\right) \in\right.$ 
$\left.\{0,1\}^{\omega}:\left(x_{1}, \ldots, x_{k}\right)=\left(j_{1}, \ldots, j_{k}\right)\right\}$, and define $h: X \rightarrow X$ by

$$
h\left(\left(x_{n}\right)\right)= \begin{cases}\left(0, x_{1}, x_{2}, x_{3}, \ldots\right), & \left(x_{n}\right) \in X_{00}, \\ \left(0,0,1, x_{4}, x_{5}, x_{6}, \ldots\right), & \left(x_{n}\right) \in X_{010}, \\ \left(0,1, x_{4}, x_{5}, x_{6}, \ldots\right), & \left(x_{n}\right) \in X_{011}, \\ \left(x_{1}, x_{2}, x_{3}, \ldots\right), & \left(x_{n}\right) \in X_{1} .\end{cases}
$$

The following diagram illustrates the action of $h$ :
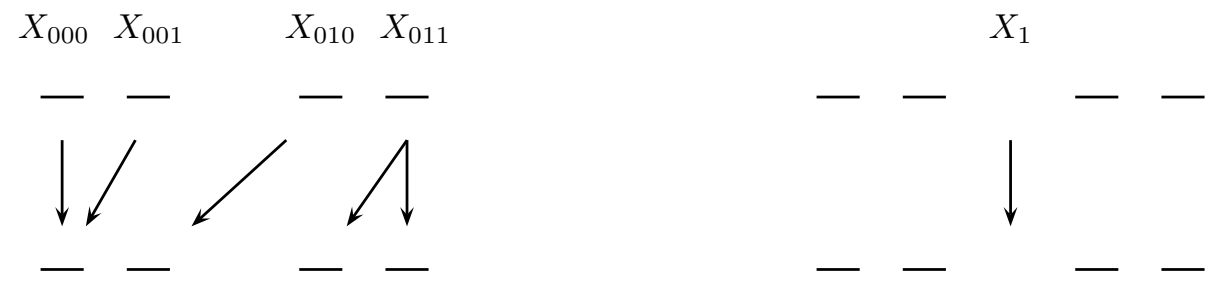

Obviously $h$ sends $X_{00}$ into $X_{000}$ and is the identity on $X_{1}$, and one checks easily that $h$ is a self-homeomorphism of $X$. Furthermore, the $n^{\text {th }}$ iterate $h^{n}$ sends $X_{00}$ into the set $X_{0 \cdots 0}$ consisting of elements of $\{0,1\}^{\omega}$ that begin with $n+2$ zeros. Thus taking $U_{p}=X_{00}$ and $U_{q}=X_{1}$, we see that condition (ii) of Lemma 5.4 holds.

The above proof can be easily modified to show that Theorem 5.3 holds with the Cantor set replaced by any Cantor space (that is, any space of the form $\{0,1\}^{J}$ where $J$ is an arbitrary index set).

\section{REFERENCES}

[1] Herbert Alexander and John Wermer, Several complex variables and Banach algebras, 3rd ed., Graduate Texts in Mathematics, vol. 35, Springer-Verlag, New York, 1998. MR 1482798 (98g:32002)

[2] H. S. Bear, Complex function algebras, Trans. Amer. Math. Soc. 90 (1959), 383-393. MR.0107164 (21 \#5889)

[3] A. S. Besicovitch, Totally heterogeneous continua, Proc. Cambridge Philos. Soc. 41 (1945), 96-103. MR0013764 (7,195d)

[4] Andrew Browder, Introduction to function algebras, W. A. Benjamin, Inc., New YorkAmsterdam, 1969. MR.0246125 (39 \#7431)

[5] J. de Groot and R. J. Wille, Rigid continua and topological group-pictures, Arch. Math. 9 (1958), 441-446. MR0101514 (21 \#324)

[6] T. W. Gamelin, Uniform Algebras, 2nd ed., Chelsea Publishing Company, New York, NY, 1984.

[7] Joan E. Hart and Kenneth Kunen, Complex function algebras and removable spaces, Topology Appl. 153 (2006), no. 13, 2241-2259, DOI 10.1016/j.topol.2004.09.012. MR2238728 (2007d:46044)

[8] L. Hörmander and J. Wermer, Uniform approximation on compact sets in $C^{n}$, Math. Scand. 23 (1968), 5-21 (1969). MR0254275 (40 \#7484)

[9] Alexander J. Izzo, Uniform algebras on the sphere invariant under group actions, Math. Ann. 344 (2009), no. 4, 989-995, DOI 10.1007/s00208-009-0349-1. MR2507636 (2010f:46079)

[10] Alexander J. Izzo, Uniform algebras invariant under transitive group actions, Indiana Univ. Math. J. 59 (2010), no. 2, 417-426, DOI 10.1512/iumj.2010.59.4032. MR2648073 (2012a:46089)

[11] Alexander J. Izzo, Uniform approximation on manifolds, Ann. of Math. (2) 174 (2011), no. 1, 55-73, DOI 10.4007/annals.2011.174.1.2. MR2811594(2012f:46087) 
[12] Kenneth Kunen, The complex Stone-Weierstrass property, Fund. Math. 182 (2004), no. 2, 151-167, DOI 10.4064/fm182-2-5. MR2100625 (2005k:54025)

[13] Kenneth Kunen, Ordered spaces, metric preimages, and function algebras, Topology Appl. 156 (2009), no. 7, 1199-1215, DOI 10.1016/j.topol.2008.12.023. MR2501996 (2010a:54027)

[14] Walter Rudin, Subalgebras of spaces of continuous functions, Proc. Amer. Math. Soc. 7 (1956), 825-830. MR0082650 (18,587a)

[15] Walter Rudin, Continuous functions on compact spaces without perfect subsets, Proc. Amer. Math. Soc. 8 (1957), 39-42. MR0085475(19,46b)

Department of Mathematics and Statistics, Bowling Green State University, BowlING Green, Ohio 43403

E-mail address: aizzo@math.bgsu.edu 